\section{D-Mannose schützt vor Harnwegsinfekten}

\section{Originalpublikation}

Kranjčec B et al (2013) D-mannose powder for prophylaxis of recurrent urinary tract infections in women: a randomized clinical trial. World J Urol doi: 10.1007/ s00345-013-1091-6

Eine kontrollierte Studie bestätigt: D-Mannose eignet sich ebenso gut wie das Antibiotikum Nitrofurantoin zur Prophylaxe von Harnwegsinfekten bei Frauen, hat aber weniger Nebenwirkungen.

Der Zucker D-Mannose wird bereits recht häufig zur Prophylaxe von Harnwegsinfekten bei Frauen verwendet, allerdings ist die Studienlage zum Nutzen der Substanz noch eher dünn, berichten Ärzte um Bojanna Kranjčec von der Klinik in Zabok in Kroatien. Die Urologen um Kranjčec haben nun die Wirksamkeit des Zuckers mit der des Prophylaktikums Nitrofurantoin verglichen. Dazu behandelten sie Frauen mit einer akuten Harnwegsinfektion und einer Historie von Rezidiven zunächst sieben Tage mit Ciprofloxacin. Danach wurden die 308 Teilnehmerinnen in drei Gruppen eingeteilt.

Etwa ein Drittel erhielt zur Prophylaxe weiterer Infektionen ein halbes Jahr lang D-Mannose $(2 \mathrm{~g} / \mathrm{d}$ in $200 \mathrm{ml}$ Wasser), ein Drittel bekam 50 mg/d Nitrofurantoin und beim letzten Drittel

Urologe $2013 \cdot 52: 1134$

DOI 10.1007/s00120-013-3274-6

(c) Springer-Verlag

Berlin Heidelberg 2013

Redaktion

H. Rübben, Essen wurde auf eine medikamentöse Prophylaxe verzichtet.

\section{Rezidivrate um drei Viertel reduziert}

Nach sechs Monaten war es bei 98 Patientinnen (32\%) zu Rezidiven gekommen. Bei allen Rezidivpatientinnen erfolgte ein Erregernachweis. In der Gruppe ohne Prophylaxe hatten mehr als die Hälfte der Frauen Rezidive erlitten (61\%), dagegen waren es mit D-Mannose nur 15 Frauen (15\%) und mit Nitrofurantoin 21 Frauen (20\%).

In beiden aktiven Prophylaxegruppen war die Rezidivrate im Vergleich zur Kontrollgruppe signifikant reduziert - um $76 \%$ mit D-Mannose und um 66\% mit dem Antibiotikum. Die Unterschiede zwischen den beiden geprüften Substanzen bei der Wirksamkeit waren jedoch nicht signifikant.

Allerdings war die Rate unerwünschter Wirkungen mit dem Zucker im Vergleich zu der mit dem Antibiotikum deutlich geringer. Nur acht Frauen mit D-Mannose, aber 29 mit Nitrofurantoin klagten über Nebenwirkungen, am häufigsten in beiden Gruppen war Durchfall (8-10\%). Patientinnen mit Nitrofurantoin nannten zudem noch Kopfschmerz, Rush und vaginales Brennen. Insgesamt seien aber beide Substanzen so gut vertragen wurden, dass es keine Therapieabbrüche wegen Nebenwirkungen gab, schreiben die kroatischen Urologen.

D-Mannose ist für die Glykosylierung eine Reihe von Proteinen nötig. Es bindet aber auch an Pili von Enterobakterien und verhindert damit, dass die Keime ans Uroepithel andocken.

Thomas Müller www.springermedizin.de

\title{
Gebräuchliche Schmerzmittel erhöhen
} Nierenkrebsrisiko

\section{Originalpublikation}

Choueiri TK et al (2013) Analgesic use and the risk of kidney cancer: A meta-analysis of epidemiologic studies. Int J Cancer doi: 10.1002/ ijc.28093

Immer mehr Studien attestieren insbesondere Acetylsalicylsäure (ASS), aber auch (anderen) NSAR eine krebsvorbeugende Wirkung, unter anderem gegenüber Karzinomen des Kolorektums, der Brust und der Prostata. Ein Schutz vor Nierenkrebs lässt sich jedoch nicht feststellen. Die meisten Analgetika scheinen das Erkrankungsrisiko sogar zu fördern, wie eine Metaanalyse jetzt ergeben hat

Die Analyse fußt auf Daten von zwölf Fall-Kontroll-Studien mit 7.075 Erkrankten und 579.285 Kontrollen und acht Kohortenstudien mit 1.165 Erkrankten unter 579.285 Teilnehmern. Danach ist die gelegentliche oder regelmäßige Anwendung von Paracetamol mit einem Anstieg des Nierenkrebsrisiko um $28 \%$ verbunden. Höhere Dosierungen gingen mit einem höheren Risiko einher (plus $68 \%$ ). Anwender von NSAR ohne ASS hatten eine um $25 \%$ erhöhte Nierenkrebsrate.

Auch hier zeigte sich eine Dosis-Wirkungs-Beziehung mit einer Steigerung der Krebsrate um $56 \%$ bei Einnahme hoher Dosen. Wurden nur Studien verwendet, in denen eine Adjustierung für andere Risikofaktoren für Nierenkrebs, nämlich Übergewicht und Rauchen, stattgefunden hatte, dann war der Zusammenhang zwischen jeglicher Behandlung mit Paracetamol oder NSAR und Nierenkrebs sogar noch deutlicher, mit einer Steigerung um $23 \%$ be- ziehungsweise $38 \%$. ASS hatte in 13 Studien insgesamt keinen Einfluss auf die Nierenkrebsrate. Auch die Anwendung von höheren Dosierungen oder über längere Zeit steigerte das Risiko nicht

Dr. Beate Schumacher Uro-News 2013; 17(5):8 